\title{
La trouvaille de monnaies carolingiennes de Zelzate
}

Le 17 novembre 1949, des ouvriers, creusant sous la direction du cantonnier Lefebure, un fossé le long de la route d'état ErtveldeZelzate, à gauche de la distance 15.000, au hameau "De Catte", sur le territoire de la commune de Zelzate (Flandre Orientale), rencontrèrent un corps dur qu'ils prirent d'abord pour une mine, et situé à une profondeur de 0,30 à $0,40 \mathrm{~m}$. sons le niveau actuel. Déterré avec précaution, l'objet se révéla être une cruche de terre cuite, placée verticalement, la base en haut, et nc présentant ni bouchon ni autre fermeture. Quand on souleva la cruche, des monnaies s'en échappèrent en même temps que des matières végétales de teinte grisâtre.

Disons quelques mots de l'endroit où ce trésor fut découvert. Zelzate est une conmmune dont le territoire appartenait primitivement à la paroisse et "métier " d'Assenede. Le métier d'Assenede a du certainement faire partie d'un ensemble d'iles et d'ilots, de "schorren" ou terres périodiquement inondées par la mer, situées dans l'embouchure de l'Escaut Occidental ou Honte. Plus tard, ces terres se sont progressivement soudées à la terre ferme. On ne connaissait jusqưa présent de mention d'Assenede antérieure à 1108 , et la trouvaille de Zelzate est donc déjà intéressante en ce qu'elle prouve que l'endroit était probablement émergé au milieu du $9^{\circ}$ siècle.

Il n'est pas sans intérêt de rappeler que le Métier d'Assenede ressortait au point de vue ecclésiastique, non à l'évêché de Tournai comme la terre ferme au nord de Gand, mais à celui d'Utrecht, sans doute parce qu'Assenede était séparé encore de la terre ferme au moment où les limites des évêchés furent déterminés. Assenede appartenait dailleurs, sans doute pour la même raison, non au royaume de France, mais à l'Empire. A un autre point de vue, il est évident que ce territoire appartenait à celui des "Frisons" tel qu'il est délimité par la Lex Frisionum du début du $9^{\text {e }}$ siècle.

Quelques indications, nécessairement très vagues, sur. l'emplacement exact de la trouvaille: l'endroit est situé au hameau De'Catte, le long de la route Meulestede-Zelzate, à très peu de distance de la frontière hollandaise, et du Poeldijk. A peu de profondeur le sol est dur, "rotsgrond" nous déclare le cantonnier, et c'est sur cette couche que reposait la cruche.

A moins que cette dernière n'ait été apportée par la mer ou par un naufrage, il faut donc admettre que l'endroit était émergé à l'époque ou le trésor fut confié au sol. Le Poeldijk aurait-il déja 
existé à ce moment? Certainement non, il date du $17^{\text {e }}$ siècle et d'ailleurs, les premières traces d'endiguements ne remontant pas au dela de l'extrême fin du $10^{\text {e }}$ siècle. Il est cependant certain que l'emplacement de la trouvaille, s'il n'était pas submergé, était tout proche de la mer. Quant à la route Meulestede-Zelzate, impossible de déterminer son âge. Elle pourrait être extrêmement ancienne puisqu'il existait une route antérieure à la naissance de la ville de Gand et qui, venue de Courtrai et d'au dela, quitte la ville en direction de Meulestede et se dirige selon toute apparence vers le Nord. Mais alors, Assenede aurait déjà du être soudé à la terre ferme à une époque très reculée et on ne comprendrait plus qu'elle ait été rattachée au diocèse d'Utrecht.

En résumé, le "trésor de Zelzate " a très probablement été enfoui dans ce qui était alors une petite ile de l'embouchure de l'Escaut, en pays de droit frison.

La trouvaille comprend donc une cruche et un certain nombre de monnaies ${ }^{1}$. Le nombre de ces dernières était d'environ 450 , chiffre qui, s'il n'est peut-être pas absolument exact ne peut être erroné que de quelques unités au plus. Des contestations surgirent relativement à la propriété de ce trésor. Elles sont loin d'être apaisées aujourd'hui. Au moment où nous écrivons, le trésor se trouve dispersé en de nombreuses mains, mais essentiellement en quatre lots d'importance inégale. Le plus important, 235 pièces, se trouve en dépôt à l'administration des Ponts et. Chaussées où nous avons pu l'étudier à loisir grâce à l'extrême complaisance de $\mathrm{M}$. le directeur Colle. Un second lot, lequel primitivement comportait une centaine de pièces, mais se trouve aujourd'hui dispersé, a été reconstitué en partie ( 81 pièces) pour nous par son propriétaire primitif, $M$. Hesters. Un troisième lot, numériquement aussi important que le précédent, est déténu par un M. Van Hoecke, d'Assenede, qui a obstinément refusé de nous en laisser prendre connaissance. Cette attitude gratuitement antiscientifique montre une fois de plus combien il importe d'empêcher que le premier venu dispose de trouvailles d'importance scientifique, sur lesquels l'état doit avoir un droit de préemption. Un quatrième lot de 21 pièces se trouve aux mains de $M$. le juge de paix de Zelzate et de son greffier. Nous avons pu aussi exa-

(I) Voici l'indication exacte de quelques travaux cités souvent en abrégé dans les pages ci-dessous :

M. Prou, Les monnaies carolingiennes (Catalogue des monnaies françaises de la Bibliothèque Nationale) Paris, 1896 .

A. Enger \& R. SerRURe, Traité de numismatiqule du moyen-age, t. I. Paris, I89I.

E. GARIEL, Les monnaies royales de France sous la race carolingienne, t. I., Strasbourg, I883.

P. C. J. A. BOELES, Les trouvailles de monnaies carolingiennes dans les Pays-Bas, spécialement celles des trois provinces septentrionales (Jaarboek van het Koninklijk Nederlandsch Genootschap voor Munt en Penningkunde te Amsterdam, II), rgr5.

A. Blanchet \& A. Difunonnk, Manuel de numismatique française I, Paris 1912 . 
miner ces pièces. Les quelques monnaies restantes enfin, sont dispersées entre plusieurs mains. De celles-là encore, nous avons pu en examiner cinq. De ce qui précède, il résulte donc que nous avons eu en mains 342 pièces sur un total de 450 , soit les trois quarts. A moins que' les pièces détenues par $M$. Van Hoecke nè constituent un choix de pièces rares, nous avons donc pu prendre connaissance d'un pourcentage de pièces suffisant et au delà pour nous permettre de tirer des conclusions solides ${ }^{2}$.

Parlons d'abord de la cruche. C'est une gourde plate et intacte en poterie pcinte, mesurant $0,22 \mathrm{~m}$. en hauteur, $0,20 \mathrm{~m}$. en largeur, et $0,13 \mathrm{~m}$. à son petit axe en coupe horizontale.

Son contour presque circulaire se perd insensiblement dans un col court et cylindrique. De chaque côté de ce col sont appliquées deux petites anses plates à rebords aigus. La pâte se compose de terre glaise très pure, mélangéc à du sable. La surface offre une teinte qui varie du brun très pâle à un jaune-orange très pâle. Elle est terne et montre de fines granulations.

Les deux faces de cette gourde sont décorées de lignes plus ou moins centrifugales, exécutées grossièrement à la brosse, accompagnées de quelques éclaboussures. La teinte de cette décoration est, irrégulière et va du brun orange foncé au brun noir. Elle a été obtenue au moyen d'un lait de terre glaise très ferrugineuse, oxydé par la cuisson ${ }^{3}$. La couche de cette peinture a une épaisseur inégale et est empâtée par endroits. D'autre part, l'on aperçoit des.traces du tour du potier tant à l'intérieur du vase, aux deux faces, qu'à la face externe la moins bombée (voir dessin ci-joint).

On connaît cette forme de poterie depuis l'antiquité et elle prolonge son existence jusqu'à l'époque moderne ${ }^{5}$. Notre cruche appartient au genre dit Pingsdorf, qui doit son nom à la découverte d'un four de potier ainsi que de multiples débris de vases de ce genre

(2) Bien de personnes nous ont apportées leur aide précieuse au cours de nos recherches relatives à ces monnaies. Citons tout particulièrement $M$. Colle, directeur à l'Adninistration des Ponts et Chaussées, dont la complaisance s'est avérée inépuisable, M. l'échevin Vermeulen, qui nous a prêté une aide efficace et appréciée; n’oublions pas de citer le cantonnier Lefebure, dont les indications nous ont été très utiles. Nous remetcions ici tout spécialement $\mathrm{Mr}$ le professeur Coremans qui a bien voulu se charger de l'analyse chimique de la peinture de la cruche.

(3) L'analyse chimique de cette peinture a été faite au Laboratoire Central des Musées de Belgiquie A.C.L. à Bruxelles sous la direction du Prof. P. Coremans. Ref. L.2/27006/RS/RB.

(4) Cf. C. F. A. SchaEFrer, Missions de Ras Shamra, $t$. III, Ugaritica, París, 1939, fig. $46 \mathrm{a}$, du XV-XIVe siècle avant notre ère. W. ANDREe, Die Parthenstadt Assur, Leipzig 1943, pl. $47 \mathrm{C}$ (époque parthe); M. J. Morgan, Mémoires de la délégation perse, t. VIII, Paris I905, p. 65, fig. 76 de Tepe Moussian : signalons un type analogue (d'epoque franque) trouvé à Engers (Rhénanie). Publié par F. OrLmanN, Bericht über die Tätigkeit des Landesmuseums in Bonn, BONNER JAHRBURCHER, 142, 1937, p. 198, pl. 57,2.

(5) Voir, par exemple, at Musée de la Byloke à Gand, les gourdes en „steinzeng", importées de la Rhénanie. 
fait à Pingsdorf, près de Cologne, en 1897, par C. Koenen : On en a retrouvé des exemplaires en Rhénanie ${ }^{7}$, aux Pays-Bas ${ }^{8}$, en Belgique ${ }^{\circ}$ et dans le Nord de la France ${ }^{10}$. Durant plusieurs siècles la poterie courante dans cette partie de l'Europe aurait été de ce type. Une étude d'ensemble traitant de cette céramique fait néanmoins défaut jusqu'à présent.

Les auteurs sont divisés au sujet de la date de son apparition. D'après l'Allemand Loeschke elle ne serait pas antérieure aux 12 $13^{\circ}$ siècles ${ }^{11}$, tandis qu'au contraire l'abbé Cochet estime les exemplaires "les plus anciens de l'époque capétienne et même se rattachant un peu aux derniers temps de la période carolingienne ${ }^{12}$." $\mathrm{J}$. H. Holwerda place son apparition à la fin du $\mathrm{IX}^{\circ}$ ou au début du $\mathrm{X}^{\mathrm{e}}$ siècle ${ }^{13}$, cependant que d'autres spécialistes hollandais, se basant comme Holwerda sur les trouvailles de Duurstede, où on rencontre quelques rares tessons du type Pingdorf parmi un grand nombre de fragments de poterie plus ancienne, placent maintenant les débuts de ce type vers $850^{14}$.

Il est évident qu'un type donné de céramique n'apparaitra pas tout à fait à la même époque dans des régions différentes, mais il est douteux que l'écart soit très grand en l'espèce, l'aire de dispersion de la poterie de Pingdorf n'étant pas très étendue et formant une zone de passage et d'intercours fréquent.

La trouvaille de Zelzate permet de trancher décisivement la controverse sur la date de l'apparition du type de Pingsdorf : ainsi qu'on le verra plus loin, les monnaies contenues dans la cruche indiquent que cette dernière a du être confiée au sol en ou très peu de temps après 843. L'exemplaire de Zelzate constitue donc le spécimen daté le plus ancien. Il apparait par conséquent que ceux qui attribuaient

(6) C. KOENEN, Karlingisch-fränkische. Töpfereien bei Pingsdorf, BONNEK JAHRBUECHER, IO3, 1898 .

(7) Cf. note précédente et M. LUECKGER, Trierer Zeitschrift, 1933, p. $4 \mathrm{I}$ sq. Voir aussi F. OrLmanN, op. cit., p. 256 sqq., les trouvailles de Unterbörsch et de Liblar.

(8) Notamment à Burgh, Schouwen, Egmond, Walcheren, Katwijk-Binnen, Loo, Montferland, Oostmarsum, Uddelermeer, etc. Cf. W. C. BRAAT, Middeleeuwsch aardewerk, dans GeDinkBoek van GrHFe, Meppel 1947, pp. 46I sq.; BRAAT, Funde Mittelalterlicher Keramik in Holland, BONNER JAHRBUCH. I42, I937; J.G.N.RfNaUd, Oud Gebruiksaardewerk, Amsterdam, 1948, pp. 90, 94, II9; J.H. HolwhRDA, Nederland's V'roegste Beschaving, Amsterdam 1925, p. I05 sqq.; P.C.J. BokL.S, Friesland tot de elfde eeuw, Leeuwarden, I927, p. 235 sqq.

(9) Cf. DE Lor, Bclgique Ancienne, catalogue descriptif et raisonne, Brux. I929, t. IV, pp. I78-18r.

(ro) Abbé B. Cochrt, Sépultures gauloises, romaines, franques et normandes, Paris, 1957, p. 352:

(II) S. Lorschke, Jahresberichte Prov..Mus. Trier, 1916/7, p. 6i sqq. \& pl. IX a.

(12) Abbé Cochet, op. cit., loc. cit.

(13) DORLSTAD EN ONZE VROEGSTE MIDDELLEUWE, Leiden, s.d., p. 60, 6r, 62 ; du même, OUdhink. MEDED. RIJKSMUSEUM OUDHEDHN LEIDEN, N.V.R. I, Leiden, '1924, p. 45 ; du même, NkDkrt. VR. GeSchind. loc. cit.

(14) Voir notamment les auteurs cités ci-dessus à la note 8 . 


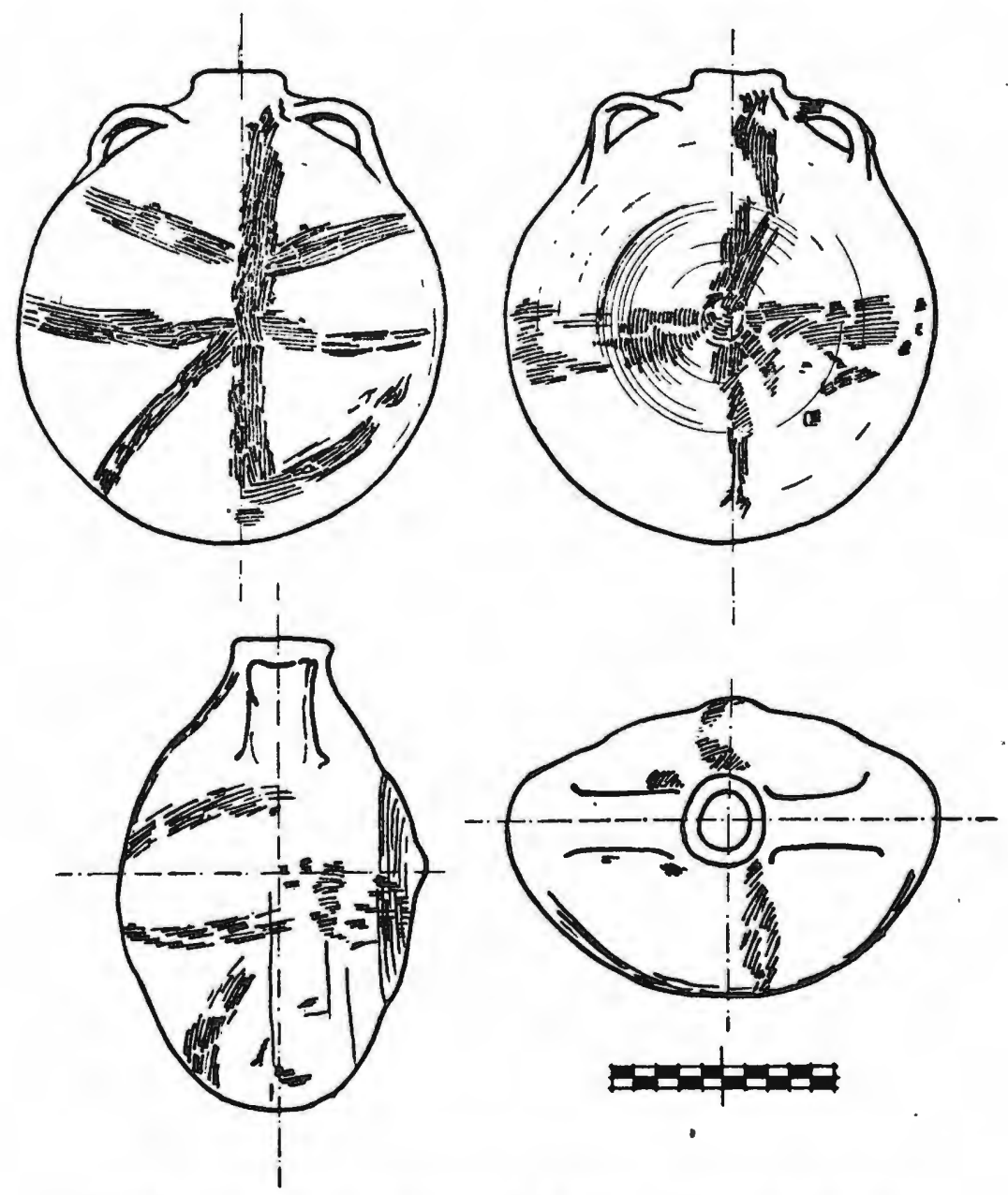

Gourde carolingienne trouvée à Zelzate. Différents aspects.

à cette poterie une origine remontant au milieu du $9^{\circ}$ siècle au moins avaient raison. La cruche de Zelzate offre bien par ailleurs les caractéristiques des plus anciens exemplaires de ce genre de céramique: sa décoration par lignes centrifugales est analogue aux exemplaires publiés par W. C. Braet il y a quelques années ${ }^{15}$ et elle fait penser à une imitation de celle des amphores à bande en relief de l'époque carolingienne.

Par ailleurs, la présence de traces du tour de potier distingue la cruche de Zelzate des produits céramiques de l'époque précédente,

(15) Middeleguwsch Aardewerk, pl. 22, $\mathrm{n}^{\circ}$ 2; Mitrelaliterl. Karamak, p. 160, pl. 2. 
ou ces traces étaient soigneusement dissimulées, et prélude à l'époque suivante ou l'on va exploiter cette technique a des fins décoratives ${ }^{18}$.

Procèdons maintenant à l'examen des monnaies.

De loin les plus nombreuses sont les monnaies très communes de Louis le Pieux au type du temple, Ce sont les deniers d'argent bien connus portant à l'avers HLUDOVVICUS IMP en légende circulaire, entre deux grenetis et dans le champ une croix cantonnée de quatre globules. Le revers porte un temple tetrastyle surmonté d'une crcix, et l'inscription XPISTIANA RELIGIO en légende circulaire.

De ces pièces, le trésor en contenait au moins 288 (sur 342 pièces examinées). Si, comme il est pratiquement certain, la proportion de pièces de ce type était la même dans la partie du trésor que nous n'avons pu examiner, les huit dixièmes étaient des deniers communs de Louis le Débonnaire.

On sait que ces monnaies offrent parfois des variantes dont le sens est obscur. Voici celles que nous avons relevées:

a. 1 exemplaire ne contient pas de croix dans le temple, mais offre 5 colonnes au lieu de 4 .

b. Un exemplaire possède bien la croix, mais il s'agit de 5 colonnes dont celle du milieu a été transformée en croix par une barre horizontale.

c. Au moins 10 exemplaires contiennent une croix de même hauteur que les colonnes du temple. Plusieurs de ces exemplaires portent en outre trois points $\left({ }_{* *}^{*}\right)$ sous la base du temple.

d. 1 exemplaire porte un point sous la base du temple.

c. 1 exemplaire porte un point sous la base du templc, et en outre un point à gauche et à droite du temple.

f. I exemplaire porte un point unique à gauche du temple.

g. i exemplaire porte un petit demi cercle ouvert vers le haut sous la base du temple.

h. 4 exemplaires portent un $\mathrm{V}$ ouvert vers le haut sous la base $\mathrm{du}$ temple.

i. Un autre exemplaire offre la même particularité; en outre, tous les caractères de l'avers et du revers sont inversés.

Il est à peine besoin de dire qu'il y a des variantes dans la graphie de XPISTIANA RELIGIO. Nous ne les relevons pas ici. Pour ce qui est du poids de ces deniers, il varie considérablement. Sur 37 exemplaires pèsés, le plus lourd avait un poids de 1 gr 75 , le plus léger 1,33. 20 d'entre eux pèsent au moins $1,60 \mathrm{gr}$.

(16) La céramique de Pingsdorf demeura en usage, pendant plusieurs siècles et vraisemblablement jusque vers les années 1300 (cf. RENAUD, $o p$. cit. p. IIg). Notons que la cátramique du type de Pingsdorf, mais sensiblement plus évoluée que celle de Zelzate, a été trouvée lors des fouilles du castellum d'Ename sur l'Escaut, dont le site temonte à l'époque 975-1050. Ce fait constitue un second repère chronologique. 
Outre ces nombreux deniers, le trésor comprenait une seule obole, absolument identique au denier sauf pour la dimension: elle pèse, $0,65 \mathrm{gr}$.

Après les monnaies au temple de Louis le Pieux, mais très loin en arrière, les pièces de Charles le Chauve au type du temple sont les plus nombreuses: en groupant trois types de variantes, on aboutit à 18 deniers et une obole. 15 deniers correspondent parfaitement au type décrit par Prou sous le $n^{\circ}$ 1057. (CARLUS REX FR entre deux grenetis, croix cantonnée de quatre globules. Revers: XPISTIANA RELIGIO, temple tetrastyle sur deux degrés, fronton triangulaire surmonté d'une croix, au centre du temple une croix, grenetis au pourtour). Quatre autres offrent la graphie CURLUS (soit l'A renversé). Un treizième denier est du type Prou no 1062 (RELCIO au lieu de RELIGIO). Deux autres deniers enfin offrent les variantes KARLUS REX FR et au revers DELICIO. Ces deniers de Charles le Chauve sont notablement plus légers que les pièces de Louis le Pieux: 8 exemplaires pèsés donnèrent pour poids $1,61 \mathrm{gr}, 1,54 \mathrm{gr}, 1,53$ gr, 1,52 gr (deux), 1,481/2 gr, 1,40 gr (deux) ${ }^{17}$.

L'unique obole est, sauf par la dimension (poids 0,72 gr.) totalement identique aux deniers du type Prou $\mathrm{n}^{\circ} 1057$.

Par importance numérique, ce sont les pièces de l'empereur. Lothaire, du type Prou $n^{\circ} 1051$ et apparentés, qui viennent en troisième rang. Nous en avons vu 11 exemplaires avec quatre variantes: 6 correspondent totalement au type 1052. (+ HLOTARIUS IMP entre deux grenetis; croix cantonnée de quatre globules. Revers temple du type ordinaire et XPISTIANA RELIGIO). Un septième est identique au précédent, sauf par la graphie LUTHARIUS. Deux autres portent HLOTARIUS IMPERATOR et les deux derniers HLOTARIUS IMPERATO, les autres éléments étant identiques au type Prou 1051. Cinq pièces de Lothaire donnent pour poids 1,$63 ; 1,55 ; 1,53 ; 1,51$ et 1,45 gr. (La pièce la plus légéré étant celle LUTHARIUS).

Viennent alors les tout petits groupes.

On rencontre d'abord les pièces de Pépin Ir d'Aquitaine : 5 oboles et 1 denier. Ce dernier, qui pèse $1,38 \mathrm{gr}$. porte en légende circulaire

(17) Soit, les conditions de conservation et de détérioriation étant identiques pour les deniers de Lonis le Pieux et ceux de Charles, une diminution dans le poids des deniers de Charles par rapport à ceux de Louis. La constatation n'aurait pas grande portée, n'était qu'elle se vérifie anssi dans le trésor d'Achlum, à peu de choses près contemporain de celui de Zelzate : selon GARIKL, Monnaies royales de France sous la race Carolingienne, I, Strasbourg $188_{3}, \mathrm{pp} .73, \mathrm{n}^{\circ} \mathrm{I}$ et 2 , vingt deniers au temple de Louis auraient un poids moyen de I,70 grammes - ce qui est tıès supérieur au poids moyen de ces monnaies à Zelzate, mais il faut tenir compte des conditions de conservation différente - et 5 deniers de Cliarles au type indiqué ci-dessus auraient un poids moyen de $1,60 \mathrm{gr}$. Les 8 deniers de CARLUS de Zelzate ont un poids moyen de I,50 gr; 36 monnaies (variantes exclues) de Louis au temple de Zelzate ont un poids moyen de $1,564 \mathrm{gr}$. Sams trop vouloir insister sur une moyenne qui repose sur trop peu de pièces pesées, nous ferons remarquer que 5 deniers HLOTARIUS IMP au temple trouvés à Zelzate donnent une moyenne de $1,53 \mathrm{gr}$. 
PIPPINUS REX + et dans le champ une croix cantonnée de quatre globules. Au revers le temple tetrastyle classique et en légende circulaire. AQUITANIORUM +. Il est donc fort proche de la figure 412 de Serrure ${ }^{18}$ et appartiendrait alors à Pépin Ir. Les oboles sont du type 659 de Prou, et donc de Pépin I ${ }^{\text {ar }}$.

Les autres "groupes" portent le nom d'ateliers urbains. Il s'agit d'abord de trois deniers de Sens, monnaies de Charles le Chauve du type Prou 574; ensuite de 2 deniers de Reims, aussi de Charles le Chauve, du type Prou 296; enfin de deux deniers de Laon. Le premier porte, entre deux grenetis, LUGDUNI CL+MONETA, et dans le champ une croix cantonnée de quatre globules. Au revers le temple tetrastyle et la devise XPISTIANA RELIGIO. Le second: identique au précédent pour ce qui est de l'avers (pour autant que les dernières lettres de l'inscription soient correctement lues; elles sont peu claires), et au revers le temple classique avec l'inscription KAROLUS GRATIA DI REX+.

Les autres pièces ne figurent à Zelzate qu'en un seul exemplaire. Mentionnons d'abord celles portant l'indication d'un atelier : il s'agit de trois deniers de Charles le Chauve : un de Mouzon (Prou $n^{\circ} 170$ ), un de l'abbaye de St Denis (Prou $n^{\circ}$ 342), et un de Quentovic (Prou $n^{\circ} 200$ ).

Viennent enfin un certain nombre de pièces sans indication d'atelier. Le premier est un denier de l'empereur Lothaire, proche du type 11 de Prou. Il porte LOTARIUS IMPIRAT + entre deux grenetis, et dans le champ une croix cantonnée de quatre globules. Au revers, le temple tetrastyle classique, et PALATINA MONETA.

Un autre denier de Lothaire, porte + HLOTARIUS ACV, entre deux grenetis. Dans le champ une croix cantonnée de 4 globules. Au revers, temple tetrastyle, XPISTIANA RELIGIO.

Les quatre pièces suivantes sont d'un roi Louis qui ne peut être que Louis le Germanique. Deux de ces deniers portent HLUDOVVICUS REX + entre deux grenetis. Dans le champ, une croix cantonnée de quatre globules. Au revers, le temple tetrastyle et XPISTIANA RELIGIO. Cette monnaie est identique, bien que d'un type plus pur, à Prou 1055.

Viennent enfin 2 autres deniers portant HLUDOVVICUS MO entre deux grenetis. Dans le champ, croix cantonnée de quatre globules. Au revers, temple tetrastyle et XPISTIANA RELIGIO.

Il y a lieu maintenant de déterminer les caractéristiques de cette collection de monnaies, afin d'arriver à établir ce qu'elle apporte de neuf pour l'histoire de la numismatique et pour l'histoire tout court.

La tâche qui s'impose tout d'abord est évidemment de replacer le "trésor" de Zelzate parmi les autres trouvailles de monnaies carolingiennes, problème qui offre un asepct chronologique, un aspect géographique et un aspect typologique.

(18) T.I.,. p. 234. L'attribution de ces monnaies à Pépin Ior est entièrement corroborée par les remarques de PROU, op. cit. p. XV, sur les légendes des pièces de Pépin : la formule EQ. manque sur nos monnaies. 
Rien de plus simple que de déterminer des termini entre lesquels ces monnaies ont été confiées au sol: cela s'est fait au plus tôt en 840, première année ou Charles le Chauve, Lothaire Ior et Louis le Germanique aient pu monnayer, cela n'a pas pu se faire sensiblement plus tard que 877 (mort de Charles le Chauve) ou même 875 (élévation de Charles à l'Empire), puisqu'aucune monnaie n'émane d'un successeur de Charles (ni d'ailleurs de ceux de Lothaire Ier et de Louis le Germanique, mais ces successeurs ont très peu monnayé, si bien que l'argument $e$ silentio ne peut être employé ici) ni de Charles le Chauve «Empereur ». Donc 840-875.

Mais il est un fait qui permet de préciser bien davantage : pour l'établir, il faudra mieux toutefois procéder à un classement chronologique des principales trouvailles de monnaies carolingiennes, du moins de celles qui, comme le trésor de Zelzate, contiennent des monnaies de Louis le Pieux, de Pépin I ou II d'Aquitaine, de Lothaire Ior et de Charles le Chauve. Ce sont des trouvailles de cette espèce que nous désignerons ci-dessous comme "contemporaines de celles de Zelzate ".

Pour donner une base plus solide à notre classement, nous débuterons par les trésors immédiatement antérieurs, c'est à dire appartenant certainement au règne de Louis le Pieux.

I. (Enfoui en 814-817). Le plus ancien est certainement celui de Veuillin (Cher). Cette antériorité ressort, comme l'a parfaitement établi Gariel (pp. 60-64 cf. p. 67) de ce que, comprenant tous les ateliers aquitains, il ne contient pourtant pas de monnaies de Pépin Ier, roi d'Aquitaine sous Louis le Pieux.

Le trésor de Veuillin est considérable: 736 pièces. Les points qui nous intéressent particulièrement sont les suivants: le trésor de Veuillin contient, comme il est naturel, un nombre considérable de pièces de la monnaie voisine de Bourges (127 deniers), mậs il contient des pièces de 36 autres ateliers, appartenant à l'Italie, la Germanie, l'Espagne et la Gaule. Relevons 18 deniers de Cambrai, 3 de Cologne, 14 de Duurstede, 2 de Mayence, 1 de Metz, 7 de Quentovic, 12 de Reims, 21 de Sens, 3 de Strasbourg, et 12 de Verdun.

Les deniers de Duurstede sont tous de Louis le Pieux, au type (Prou $\mathrm{n}^{\circ}$ 65-78) qui porte à l'avers DORESTAT en trois lignes. Ce qu'il faut relever surtout, c'est qu'aucune de ces 736 monnaies n'est au type du temple et à la légende chrétienne. Cela permet d'affirmer que Louis le Pieux n'a pas battu monnaie à ce type au début de son règne.

II. (Enfoui en 814-17, mais après I)

La trouvaille de Belvézet (Gard) appartient aussi aux début du règne de l'empereur Louis, puisqu'aucune monnaie de Pépin $I^{\text {ar }}$ n'y figure parmi les nombreux exemplaires frappés en Aquitaine. Au total, on a examiné 286 pièces; toutes, sauf 2 qui sont de Charlemagne datent du temps de Louis. Relevons 1 denier de Mayence, 
1 de. Terouanne, 1 de Verdun, 3 de Duurstede du même type qu'à Veuillin, 4 de Reims, 4 de Sens. Par ailleurs, nombreuses monnaies italiennes, espagnoles et gauloises; dans l'ensemble, 33 ateliers sont représentés.

Ce qu'il faut surtout souligner, c'est que ce trésor, enfoui dans les premières années du règne de Louis comme on l'a vu, contient un nombre, infime il est vrai ( 3 deniers, 1 obole), de monnaies au type du temple. Ce type étant, comme on va le voir, surabondant à la fín du règne, il faut admettre avec Gariel (pp. 65-68), que ce trésor est un peu postérieur au précédent.

III. (Enfoui en 840 ou aussitôt après). Négligeant la trouvaille de 32 pièces de Louis le Pieux faite près d'Angers (Gariel, pp. 68-9), qui ne nous apprend rien relativement à notre objet, nous mentionnerons celle faite dans l'Indre (Gariel, p. 68-70) et qui contient, outre 23 pièces de Louis et de Pépin d'Aquitaine, un denier de Charles le Chauve au type du temple. Elle appartient donc à l'époque immédiatement postérieure à la mort de l'empereur Louis en 840, et nous relevons trois points : a. elle contient 16 deniers (66\%) de Louis au type du temple, qui est donc devenu largement prédominant: b. 5 des monnaies portant mention d'un atelier sur 6, appartiennent au sud du royaume, ce qui permet d'affirmer que les monnaies au temple ont (aussi) été frappées dans cette région; c. enfin la pièce de Charles le Chauve permet d'affirmer que dès le début de son règne, on a frappé des monnaies portant à l'avers CARLUS REX FR. et au revers le temple te l'inscription XPISTIANA RELIGIO.

IV. Un trésor découvert à Thouars contient 47 monnaies, soit 45 deniers et une obole de Louis au type du temple, et un denier de Lothaire I $\mathrm{I}^{\text {er }}$ portant Lotarius $\mathrm{ACN}$. Nous n'oserions en déduire que l'enfouissement soit postérieur à la mort de l'empereur Louis, Lothaire ayant été associé à l'empire et ayant donc pu peut-être battre monnaie.

Les trouvailles III et IV établissent la prédominance du type au temple dans la seconde partie du règne de l'empereur Louis.

V. (Enfoui en 840 ou immédiatement après.)

A Achlum, en Frise, on a découvert un trésor de 498 deniers ${ }^{19}$. Un seul est de Charlemagne, un de Pépin I ${ }^{\text {er }}$ d'Aquitaine, un (pour Orlćans) pourrait être soit de Charlenagne, soit dc Charles le Chauve. Les autres sont de l'empereur Louis, sauf 3 deniers de l'atelier de Reims. Tous 3 portent CARLUS REX FR, autour d'une croix, et les deux derniers sont au type du temple. Comme on peut tenir pour établi (Prou p. XVIII) que les monnaies du type. du temple portant CARIUS REX ne sont pas de Charlemagne, mais

(I9) BOELES, op. cit., pp. 30-40. 
de Charles lc Chauve, il en résulte que ce trésor a été enfoui en 840 au plús tôt, mais très peu de temps après cette date, à preuve le nombre infime des monnaies de Charles le Chauve, et surtout l'absence totale de monnaies de Lothaire $\mathrm{I}^{\mathrm{ar}}$. On demeure surpris en effet de ce que, dans une collection aussi nombreuse de monnaies enfouies en Frise, on rencontre bien quelques monnaies de Charles le Chauve, mais aucune de Lothaire Ier qui règnait sur cette région. Lothaire a donc du peu ou pas battre monnaie au début de son règne, et ce trésor a donc du être enfoui très peu de temps après 840 .

Les monnaies de Louis le Pieux sont au nombre de 489 et 484 sont au type du temple. Les autres sont de Duurstede (3), Mayence (1), Strasbourg (1). Les monnaies de Duurstede sont de deux types différents : un denier est au buste et au navire (Prou 63-64). Les autres sont du même type qu'aux trouvailles I et II.

VI. (Enfoui peu de temps après la bataille de Fontenoy, 25 juin 841. ) C'est ici qu'il faut situer la trouvaille de Zelzate, ainsi que nous le montrerons plus loin ${ }^{20}$.

VII. Terp de la province de Groningue ${ }^{21}$. 63 deniers de Louis le Pieux, Lothaire Ir et Charles le Chauve. 57 de ces deniers sont de Louis et au type du temple. 1 est au type HLOTARIUS IMP, au verso, temple et inscription XPISTIANA RELIGIO. 2 sont du type que nous désignerons désormais comme Lothaire-Duurstede (Prou $\mathbf{n}^{\circ}$ 74-78) c'est à dire qu'il porte à l'avers HLOTARIUS IMP, au revers DORESTATUS MON et le temple.

(2o) C'est vraisemblablement à la même époque qu'il faut situer l'enfouissement d'un autre trésor de monnaies carolingiennes, celui trouvé à Muizen près de Malines. Nous ne le traitons pas ici comme une trouvaille indépendante, parce qu'il n'est pas clairement établi si les pièces qu'on en a examinées représentent la plus grosse partie du trésor. Cette trouvaille a été décrite (très maladroitement) dans le Bulletin des Musées royaux des arts décoratifs et industriels à Bruxelles, IIe série, $2^{\text {b }}$ année, Igog, pp. 74-76. Une étude due à $\mathbf{M}$. Heli Roosens et y consacrée sera publiée prochainement dans les Annales du Cercle archéologique de Malines. Nous devons à $M$. Roosens des renseignements détaillés sur les monnaies de Muizen, et lui exprimons ici toute notre reconnaissance pour ses précieuses indications. Ce trésor contient 73 monnaies (du moins, tel est le nombre des pièces que l'on 2 pu récupérer), dont un dirhem arabe. Les autres pièces sont 50 monnaies au temple de Louis le Pieux, 4 oboles de Pépin I ${ }^{\text {or }}$ d'Aquitaine, 13 deniers de Charles le Chauve un même type que celles trouvées à Zelzate, I denier au buste de Lothaire Iar, avec 1'inscription LOTARIUS REX AUGUSTUS (donc vraisemblablement antérieure à 1'époque ou Lothaire a succédé effectivement à son père) et enfin 2 oboles de Charles le Chauve pour l'Aquitaine et 2 deniers du même souverain pour Quentovic, en tous points identiques à celui de Zelzate. Bref, sauf le dirhem, la pièce de Lothaire et les 2 oboles de Charles le Chauve, des monnaies que l'on a trouvées à Zelzate et sensiblement dans les mêmes proportions; il semble danc bien que ce trésor ait été enfoui à la même époque, et il convient de déduire du trésor de Muizen que Charles le Chauve a du battre monnaie en Aquitaine très tôt après son avènement.

(21) BOELIS, op. cit., pp. 79-82. 
Deux autres monnaies de Lothaire font mention de l'atelier de Verdun et de celui de Cologne. L'unique monnaie de Charles le Chauve fait mention de l'atelier d'Auxerre, elle est du type CAROLUS REX FR, et au temple. Si nous classons cette trouvaille avant les suivantes, c'est parce qu'elle contient encore un nombre écrasant de monnaies de Louis au temple, comparativement aux pièces du type Lothaire-Duurstede.

VIII-IX-X-Xl-XII-XIII. Il convient encore de mentionner 6 autres trouvailles faites en Frise, parce qu'elles contiennent exclusivement des monnaies de Louis le Pieux, Lothaire Ier, Charles le Chauve et Pépin, comme la trouvaille de Zelzate, mais nous n'osons pas procéder à un classement chronologique entre ces trouvailles. Toutes contiennent un pourcentage considérable de monnaies de Louis le Pieux au temple, et de monnaies du type Lothaire-Duurstede, comme il ressort du tableau ci dessous :

Oudwoude ${ }^{21}$ bis

Pingjum I 22

Pingjum II ${ }^{23}$

Aalsum 24

Emmen ${ }^{25}$

Midlaren ${ }^{26}$

$\begin{array}{rcc}\text { Total } & \text { Louis au temple } & \text { Loth. Duurstede } \\ 62 & 28 & 30 \\ 173 & 101 & 58 \\ 52 & 19 & 16 \\ 48 & 22 & 24 \\ 341 & 219 & 79 \\ 85 & 28 & 48\end{array}$

Les autres monnaies d'Oudwoude sont un denier du type LOTHARIUS IMP, au revers temple et inscription chrétienne; CARLUS REX FR au temple et linscription chrétienne, et du même roi, un denier de Paris et un d'Orléans. Pingjum I contient en outre: trois Lothaire et 9 CARLUS comme à Oudwoude, 2 monnaies de Reims de ce même roi.

Pingium II contient en outre 1 denier de Pépin de Dax, un CARLUS REX FR au temple, et de ce même roi un denier de Meaux.

Aalsum contient en outre 1 denier HLOTARIUS IMP au temple et un solidus d'or de Louis ${ }^{27}$.

Emmen contient en outre 3 CARLUS REX FR d'Orléans, (deux au temple et un à la porte de ville); 11 CARLUS REX FR. sans atelier, au temple; 7 HLOTARIUS IMP au temple; 1 monnaie de Lothaire pour Huy; 1 pour Trèves; 2 pour Verdun.

Midlaren contient en outre un denier de Lothaire pour Pavie, et un denier de Charles le Chauve pour Chartres, Sens et Auxerre.

Ses données vont nous permettre de résoudre plusieurs problèmes.

(2I bis) BOELES, op. cit., pp. 79-82.

(22) Ibid. pp. 40-42.

(23) Ibid. pp. $42-45$.

(24) Ibid. pp. 44-47.

(25) Ibid. pp. 50-5i.

(26) Ibid. pp. 6I-63.

(27) Ibid. pp. 63-65. 
Le premier et le plus important est celui de la date de l'enfouissement. Nous savons déjà qu'elle est postérieure à 840 et antérieure à 875. Il apparait au premier coup d'œil que le trésor de Zelzate se caractérise par deux éléments positifs et un élément négatif. Le premier est la présence, en nombre écrasant, de monnaies de Louis le Pieux au type du temple, monnaies qui, on le sait déjà, appartien. nent à la seconde moitié du règne de l'empereur. Le second élément positif est la présence, en nombre restreint, de monnaies de Charles le Chauve, de Lothaire et d'un roi Louis qu'on a bien de la peine à ne pas identifier d'emblée au troisième des fils de Louis le P'́eux, Louis le Germanique.

L'élément négatif, c'est l'absence totale des monnaies du type Lothaire-Duurstede.

Examinons la portée de ce dernier fait: Nous connaissons, par les trouvailles I et II, le type de monnaies frappées à Duurstede au début du règne de l'empereur Louis. Elles se caractérisent par le mot DORESTATUS écrit en trois lignes. De ces monnaies nous en rencontrons encore deux exemplaires dans la trouvaille d'Achlum (V). Mais deux exemplaires (et une troisième au type du buste) sur 498, n'est ce pas extraordinairement peu, eu égard au nombre très élevé de monnaies du type Lothaire-Duurstede?

Autre renarque: nous avons souligné à propos de la trouvaille III, que des monnaies au type du temple ont été frappées dans le sud de la France.

Mais ces monnaies sont tellement nombreuses dans les trouvailles du Nord (Voyez V-XIII) qu'il faut de toute évidence admettre aussi qu'on a battu monnaie à ce type dans le Nord. Où cela ? C'est ici qu'intervient le rapport des nombres: dans les trouvailles VIII-XIII, contemporaines ì quelques anıées près des trouvailles V-VI, les monnaies de Lothaire-Duurstede sont extraordinairement nombreuses. Comment admettre que dans les années précédentes, on n'ait pas battu monnaie à Duurstede avec une égale intensité ? Et pourtant, sur les quelque mille pièces des trésors de Zelzate et Achlum combinés, on ne trouve à citer que trois monnaies portant le nom de Duurstede. Le bon sens indique qu'il faut donc chercher ces monnaies sous un autre forme, et il ne faut pas aller bien loin : où donc a-t-on frappé ces centaines de pièces au type de Louis et du temple, retrouvées dans le Nord? Que certaines proviennent d'autres ateliers, ceux du sud de la France, par exemple, c'est certain, mais n'est-il pas infiniment vraisemblable, comme l'a déjà suggéré Boeles ${ }^{28}$, que ces monnaies représentent l'abondant monnayage de Duurstede sous Louis le Pieux postulé par le monnayage surabondant de Duurstede sous Lothaire?

Que l'on admette ou non ces prémices, il demeure qu'il faut dater l'enfouissement du trésor de Zelzate d'une époque ou les monnaies du type Lothaire-Duurstede n'étaient pas encore frappées, c'est à

(28) Ibid. p. I4. 
dire en tout cas avant la mort de Lothaire en 855 , et en réalité beaucoup plus tôt, l'abondance des monnaies retrouvées indiquant qu'elles ont été frappées durant la plus grande partie du règne ${ }^{29}$.

Ces observations, qui nous amènent à situer l'enfouissement dans les années qui suivent immédiatement 840, se trouvent corroborées par deux autres données. L'une des monnaies retrouvées à Zelzate est un denier de Charles le Chauve portant le nom de l'atelier de Mouzon. Or Mouzon a fait, depuis le traité de Verdun, partie des états de Lothaire ${ }^{3 n}$. Il n'y a qu'un seul moment antérieur à ce traité où Charles a pu battre monnaie à Mouzon, c'est dans l'intervalle entre la défaite de Lothaire à Fontenoy (25 juin 841 ) (suivi de la mainmise sur ses états par les vainqueurs, Charles et son frère Louis le Germanique) et le traité de Verdun même (août 843). On ignore quelles furent les limites du partage des états de Lothaire entre les deux frères vainqueurs ${ }^{31}$, et rien ne s'oppose donc à ce que Mouzon ait appartenu à ce moment à Charles.

Une seconde et précieuse indication nous est fournie par l'existence dans le trésor de Zelzate de quatre monnaies portant le nom d'un roi Louis. Antérieurement à 855 , ce roi ne peut être que Louis le Germanique. Mais Serrure et Prou s'accordent à nier tout monnayage de Louis antérieurement à l'époque ou, en 870 , il entra en possession d'une partie de la Lotharingie ${ }^{32}$. La présence à Zelzate de monnaies de Louis prouve qu'il a bien réellement battu monnaie avant 870 , sans doute au moment même ou, après Fontenoy, il régnait sur une partie de la Lotharingie.

Ainsi donc le trésor de Zelzate comprend cinq monnaies qui ne peuvent avoir été battues qu'en 841-43. C'est là encore un indice de ce que le trésor de Zelzate a du être enfoui peu après, ces monnaies ayant du être peu nombreuses et leur présence simultanée dans une trouvaille ne s'expliquant que peu de temps après leur mise en circulation.

Mais peut-être le lecteur sera-t-il tenté de retourner contre nous ces deux arguments, et d'incliner à croire que la date d'enfouissement du trésor de Zelzate est au contraire tardive : une date qui ne serait pas antérieure à 870 expliquerait en effet, et l'existence d'une monnaie

(29) Encore faut-il remarquer que de 850 à 855 , Duurstede a appartenu au chef normand Roric (ANNales Bertiniani, ed. Waitz, p. $38, a^{\circ} 85^{\circ}$ et ibid., p. $\left.46, a^{\circ} 855\right)$. Mais comme rien n'indique que cette circonstance ait du interrompre la frappe an nom de Lothaire, il n'y a sans doute pas lieu d'en faire état ici.

(30) Le Mouzonnais est en effet mentionné dans 1'héritage de Lothaire dans le traité de Meerssen (Borerius-Krausi, Capitularia II, p. 195 I.I3).

(3I) Charles et Louis se sont en effet partagés les états de Lothaire à Aix la Chapelle, en mars 842 . Il est exact que Charles le Chauve doit traverser la Meuse pour rentrer dans ses états (NITHARD, IV-c.2), mais cela n'implique point nécessairement que ce fleuve constitue sur tout son cours la frontière, comme semblent l'admettre Lor-HALPHEN, Le règne de Charles le Chauve, p. 53, n. 6 . Il demeure en tout cas aussi que Charles a pu dominer à Mouzon entre Fontenoy et le traité d'Aix la Chapelle.

(32) Prou, p. XXI, Serrure, p. 260. 

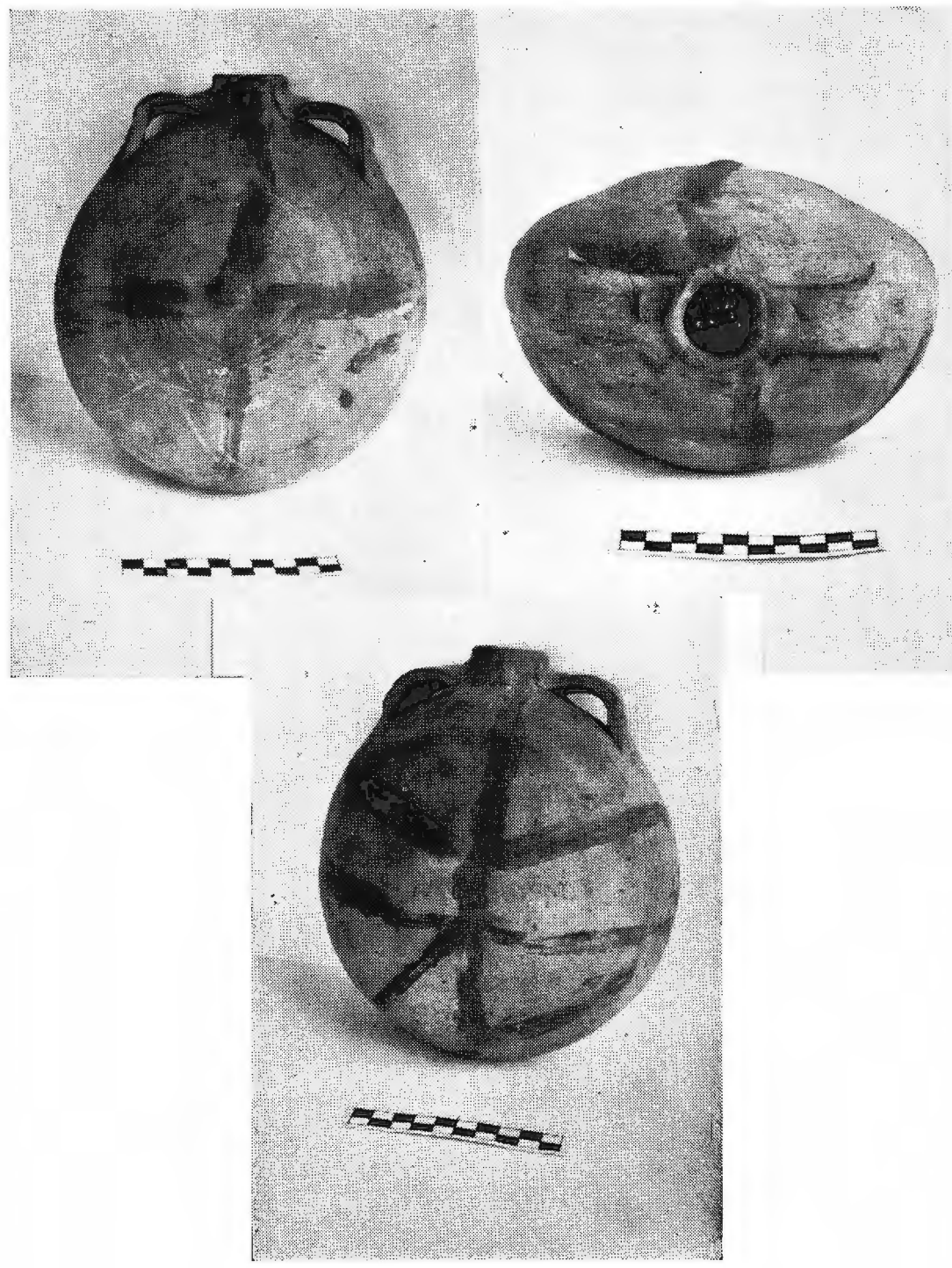

Gourde carolingienne trouvée à Zelzate.

Photographies sous différents angles. 


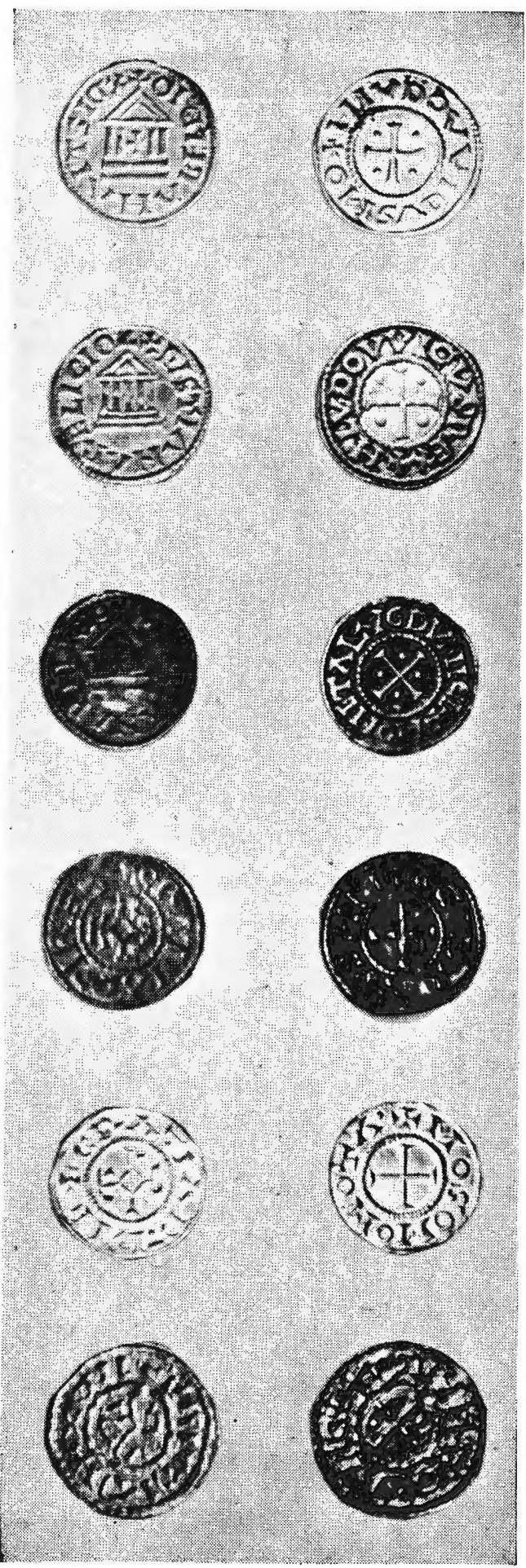

De haut en bas:

1. Louis le Germanique cf. p. 10,16

2. Louis le Germanique cf. p. 10,16

3. Charles le Chauve, Laon cf. p. 10.

4. Charles le Chauve, St Denis cf. p. 10.

5. Charles le Chauve, Mouzon cf. p. 10.

6. Charles le Chauve, Quentovic cf. p. 10. 
frappée par Charles le Chauve à Mouzon (Mouzon et une grande partie des états de Lothaire II étant passés à Charles en 870) et celle de monnaies de Louis le Germanique, la thèse généralement admise étant que ce souverain n'a battu monnaie qu'à partir du moment où il a acquis une part de la Lotharingie, soit encore en $\mathbf{8 7 0 .}$

Séduisante à première vue, cette hypothèse pourrait s'autoriser du fait, dont nous prenons connaissance au moment de mettre la dernière main à ce travail, que le trésor de Muizen, mentionné en note ci-dessus et dont la composition se rapproche très fort de celle de la trouvaille de Zelzate, contiendrait un dirhem arabe de 866. Les circonstances dans lesquelles le trésor a été en partie reconstitué n'étant pas de nature à donner toute sécurité, nous n'attacherons pas une importance exagérée à ce dernier argument, mais le raisonnement cidessus demeure valable.

Et pourtant, le trésor de Zelzate ne peut pas avoir été enfoui après ou en 870 : la preuve, c'est qu'il ne contient pas une pièce au type de Lothaire-Duurstede. Or, ces pièces sont, on l'a vu, très nombreuses dans les trouvailles frisonnes du troisième quart du siècle. A quelle époque ces monnaies ont-elles été frappées? De toute évidence, au plus tard en 855, année de la mort de Lothaire ier. L'absence intégrale de cette monnaie si répandue prouve absolument, à nos yeux, que l'enfouissement du trésor de Zelzate est de plusieurs années antérieures à 855 .

En conclusion, il ne paraît guère douteux que le trésor de Zelzate ait été enfoui à une époque qui est postérieure à la bataille de Fontenoy ( 25 juin 841 ) et bien antérieure à 855 , vu l'absence totale de deniers du type Lothaire-Duurstede, et l'abondance extrême de pièces appartenant encore au règne de Louis le Pieux. Chronologiquement, la trouvaille de Zelzate doit être de très peu postérieure à celle faite dans l'Indre (III ci dessus) et à celle d'Achlum(V). Son intérêt est de nous faire connaitre avec précision le monnayage des fils de Louis le Pieux dans les extrêmes débuts de leur règne.

Passons brièvement en revue les constatations qui s'offrent: nous soulignerons d'abord que les trois fils de Louis le Pieux ont frappé en ces premières années de leur règne, des monnaies qui ne diffèrent entre elles que par le nom du souverain: la trouvaille de Zelzate nous fait connaître les deniers de Lothaire, de Charles (il s'en trouve aussi dans les trouvailles de l'Indre et de Achlum), et de Louis, portant au revers le temple tetrastyle et la devise XRISTIANA RELIGIO, à l'avers CARLUS REX FR, HLOTARIUS IMP ou IMPERATOR, HLUDOVVICUS REX, ces légendes entre deux grenetis, dans le champ une croix cantonnée de 4 globules. C'est là la copie littérale de la monnaie au temple de Louis le Pieux, prédominante, on l'a vu, dans la seconde partie de son règne.

Le trésor de Zelzate contient encore un denier portant LOTHARIUS ACV et qui à la rigueur pourrait être antérieur à 840 et dater. de l'association de Lothaire à l'empire. Il contient aussi un exemplaire frappé à la monnaie palatine. C'est peut-être un hasard, mais 
on ne peut s'empêcher de relever qué des 13 deniers du fils ainé de Louis le Pieux, il n'en est aucun portant mention d'un atelier. Bien plus, le seul atelier "lotharingien" dont on possède une monnaie, Mouzon, a battu monnaie au nom de Charles le Chauve. Tout cela s'accorde bien avec l'existence errante que Lothaire a mené après la bataille de Fontenoy (soit après un règne effectif de un an exactement ( 20 juin 840, mort de l'empereur Louis, 25 juin 841, bataille de Fontenoy) et confirme sensiblement la thèse d'un enfouissement du trésor à une époque qui ne peut pas être très postérieure au traité de Verdun. Presque toutes les autres trouvailles frisonnes, même celle du Terp près de Groningue (VII) qui ne contient encore que deux monnaies au type Lothaire-Duurstede, comptent en effet des exemplaires ou le nom de Lothaire est accompagné de la mention d'un atelier, soit Verdun et' Cologne (VII). Huy, Trèves et Verdun (Emmen), Pavie (Midlaren).

Il est donc établi qu'au début de son règne, Lothaire a battu monnaie au type du temple sans atelier, et à la monnaie palatine.

Pour ce qui est de Louis, il a battu à deux types au moins, le style du temple pur et simple, avec HLUDOVVICUS REX, et une monnaie au temple avec HLUDOVVICUS MO. Comme il a certainement dominé en Frise et dans le nord des états de Lothaire entre Fontenoy et le traité de Verdun ${ }^{33}$, on peut se demander si le premier de ces types, identique sauf pour le nom du souverain aux monnaies que, selon la thèse proposée ci-dessus, Louis le Pieux a fait battre à Duurstedë, ne représente pas le monnayage de Duurstede à l'époque où Louis y dominait. On peut formuler la même hypothèse pour les deniers de Lothaire, pour les mêmes motifs et pour l'époque comprise entre la mort de Louis le Pieux et la bataille de Fontenoy, et éventuellement pour l'époque immédiatement postérieure au traité de Verdun. Il paraît en effet infiniment peu probable que, dans un trésor qui contiendrait une masse aussi considérable de pièces de Louis le Pieux frappées à Duurstede, il ne se trouverait pas non plus de deniers frappés à Duurstede durant les quelques années comprises entre la mort de l'empereur Louis et la date d'enfouissement du trésor. Si tout cela était avéré, on pourrait proposer le classement que voici pour les monnaies de Duurstede :

1. Au début du règne de Louis le Pieux (814-?), les monnaies retrouvées dans les trouvailles I et II ci-dessus, soit portant au revers DORESTATUS en trois lignes.

2. Dans la seconde partie du règne de cet empereur, les monnaies sans nom d'atelier, HLUDOVVICUS IMP. et légende chrétienne.

3. Entre le 20 juin 840 et le 25 juin 841 , les monnaies identiques aux précedentes, mais portant HLOTARIUS IMP.

4. Entre le 25 juin 841 et aout 843 , les monnaies identiques aux précédentes, mais portant HLODOVVICUS REX.

(33) NITHARD, IV c. I. "evenitque Lodhuvico omnis Frisia”. 
5. A partir d'un moment postérieur au traité dc Verdun, et dans un ordre que nous ne nous chargeons pas de déterminer, les deux types de deniers Lothaire-Duurstede.

Toute cette classification repose, soulignons-le, sur un postulat ou du moins une hypothèse que nous espérons avoir rendu vraisemblable, mais qui n'est pas prouvée, notamment que les monnaies au temple de Louis le Pieux, trouvées à Zelzate, à Achlum et dans le terp près de Groningue, ont été dans leur grande majorité frappées à Disurstede.

Ce sont les quelques monnaies de Charles le Chauve, trouvées à Zelzate, à Achlum (2 exemplaires) et dans l'Indre (un seul exemplaire), qui nous livrent toutefois les renseignements les plus précieux. En groupant les deniers de ces trois trouvailles, toutes enfouies, on le sait, très peu de temps après la mort de Louis le Pieux et donc dans les toutes premières années du règne de Charles le Chauve, nous aboutissons aux constatations suivantes.

Tout d'abord, il apparait que la grande majorité de ces monnaies, qui sont au total au nombre de 29 deniers et une obole, sont du type courant (CAROLUS REX FR entre deux grenetis, croix cantonnée de quatre globulcs, au revers, le temple ct la légende chrétienne). On compte 16 deniers de ce type et une obole. Beaucoup plus intéressant pour l'histoire monétaire sont toutefois les autres deniers: les deux deniers de Reims de Zelzate, et les deux deniers remois d'Achlum, sont du type Prou 296 (sauf que les pièces de Zelzate portent Carlus ct non Carolus), c'est à dire qu'elles portent à l'avers la même inscription que les pièces au temple mentionnées ci-dessus, au revers le temple et REMIS CIVITAS. Rien que de banal là dedans, sauf que les autres monnaies de Reims citées par Prou portent toutes, au lieu de CARLUS REX FR, l'inscription GRATIA DEI REX et le monogramme. L'intérêt de cette remarque, c'est qu'elle prouve que, à Reims et sans doute ailleurs, le type nouveau (GRATIA DEI REX et le monogramme) n'a été introduit qu'après un ccrtain temps, alors que, comme on va le voir plus loin, il a été introduit en certains ateliers dès le début du règne.

Les trois deniers de Sens de Zelzate (Prou 574) offrent exactement la même disposition que ceux de Reims. Mais il n'en va pas de même des deniers de Laon (du moins de l'un d'entre eux, le premier ne portant pas de nom de souverain), de Mouzon, St. Denis et Quentovic.

Les monnaies de Mouzon, St. Denis et Laon portent la devise GRATIA DEI REX. Nous voici donc désormais fixés sur l'époque où cette formule destinée à faire fortune, a été introduite sur les monnaies de Charles le Chauve.

Mais il y a plus: on trouve le monogramme de Charles le Chauve sur les monnaies de Mouzon, St. Denis et Quentovic. Et voici donc un second point controversé définitivement résolu: dès le début de son règne, Charles a introduit le monogramme ${ }^{34}$ !

(34) Prov, p. XIX, se demande, sans conclure, si ce type a été ou non in- 
En conclusion, nous voyons qu'à début de son règne Charles le Chauve a battu monnaie aux types suivants:

1. Un denier et obole qui constitue la continuation directe de la pièce de son père au nom du souverain avec le titre, dans le champ une croix cantonnée de quatre globules, au revers, le temple et l'inscription chrétienne;

2. Un denier à l'avers identique au précédent, au revers le temple et en légende circulaire, le nom de l'atelier:

3. Un denier au revers identique au précédent, mais pcrtant déjà à l'avers la nouvelle formule GRATIA DEI REX:

4. Enfin, un type de monnaie entièrement nouveau, portant à l'avers la nouvelle formule GRATIA DEI REX, et le monogramme, au revers, la croix et le nom de l'atelier en légende circulaire ${ }^{3 i}$.

Considérons encore brièvement la composition géographique du trésor de Zelzate: le gros des monnaies est, si l'on admet nos hypothèses, originaire de Duurstede. On y trouve par ailleurs, 4 oboles et un denier de Pépin Ir d'Aquitanic. Les pièces d'Aquitaine sont rares dans les trouvailles septentrionales carolingiennes. Nous en avons relevé ci-dessus l'un ou l'autre exemplaire dans notre énumération du contenu des trouvailles en Frise. Le trésor de Zelzate apparaît ici plus " méridional " que ceux de Frise.

Les monnaies de Sens n'apparaissent presque pas non plus ailleurs dans les trouvailles frisonnes (1 exemplaire à Midlaren). Celles de Reims sont en revanche assez communes: on en trouve à Achlum, dans Pingjum I, Emmen (11 exemplaires!)

Des deniers de Laon ne se retrouvent pas non plus, pas plus que ceux de Mouzon, de St. Denis ou même, chose plus surprenante, de Quentovic.

En revanche, il apparait que la trouvaille de Zelzate, relativement fournie en monnaies de France, ne contient aucune monnaie rhénane ou haut-mosane (sauf Mouzon) qui sont assez fréquentes dans les trouvailles frisonnes, même dans celle d'Achlum qui la précède chronologiquement. Il y a là une caractéristique assez nette qui distingue la trouvaille de Zelzate de celles de Frise, mais il est impossible de

troduit avant 855 . Soulignons ell revanche, que manquent totalement les monnaies à la légende CARLUS RFX autour d'une croix, avec an dos un nom de lieu écrit en deux lignes dans le champ. Fngel et Serrure les avaient attribués à Charlemagne, mais Prou (p. XVIII) les situait au début du règne de Charles le Chauve. Leur absence dans les trois trouvailles d'aussitôt après 840 n'est pas en soi un élément déterminant dans la controverse, mais ce qui ne laisse pas d'avoir une certaine importance, c'est que parmi ces monnaies il en est de Sens. Or, à moins de supposer que dans les toutes premières années du règne de Charles le Chauve on ait frappé deux types différents à la monnaie de Sens (ce qui n'est pas très probable) il faut bien tenir compte du fait que les plus anciennes monnaies de Charles le Chauve à Sens sont du type au temple, non du type au nom de lieu en deux lignes.

(35) Les monnaies de Zelzate prouvent que c'est entidrement à tort que Prou (p. XVIII) écrit que ,toutes ces pièces (de monnaies à monogramme) sont sorties d'ateliers Aquitains". 
baser une conclusion quelconque sur cette unique trouvaille. de même qu'on ne peut rien déduire de la présence de telle ou telle monnaie française dans le trésor.

Oserait-on pourtant émettre un doute sur le caractère "frison" du trésor de Zelzate? C'est à dire, pourrait-on affirmer que cette collection de monnaies serait originaire d'une toute autre partie de l'empire, et n'aurait été enfouie à Zelzate qu'accidentellement? Comme c'est là une possibilité qui théoriquement existe toujours et qu'une grande partie de nos déductions et notre chronologie postulent que le trésor de Zelzate ait été constitué dans un milieu où les types de monnaies apparaissant normalement dans les trouvailles frisonnes sont répandus, il convient d'y réfléchir.

Remarquons d'abord que Zelzate se trouvait bien dans la "Frise " carolingienne dont la limite occidentale, selon la Lex Frisionum, était le Sincfal ou Zwin près de Bruges ${ }^{36}$. Assenede, dont Zelzate n'est qu'un démembrement, appartenait à l'évêchè d'Utrecht. Il est par ailleurs de plus en plus évident que la côte flamande (Zelzate était un île ou bien se trouvait en bordure de la mer, ne l'oublions pas) appartenait à l'époque carolingienne à cet univers de la mer du Nord, très distinct du monde franc. et où les Frisons jouaient un rôle considérable. Tout cela indique qu'une trouvaille carolingienne faite à Zelzate appartient, jusqu'à preuve du contraire, au milieu frison.

La trouvaille de Zelzate offre par ailleurs une caractéristique qui la distingue de celles faites dans la moitié méridionale de la France: contrairement à ce qui est le cas à Veuillin ou à Belvezet, qui lui sont comparables par le volume, le trésor d'Assenede ne contient pas des monnaies de dizaines d'ateliers différentes, mais bien une prédominance écrasante de pièces d'un même type. C'est le cas de la trouvaille d'Achlum et de toutes les autres trouvailles frisonnes. Surtout si on admet que les monnaies de l'empereur Louis au temple, tout comme celles de Lothaire-Duurstede, sont originaires de Duurstede, le trésor de Zelzate contient presqu'exclusivement des monnaies de Duurstede. $\mathrm{Si}$ on repousse cet argument, qui constitue cn effet une pétition de principes, il demeure que la trouvaille de Zelzate contient un nombre infime de monnaies appartenant certainement à la France et qu'elle est pratiquement identique à celle d'Achlum, contemporaine et certainement frisonne.

Nous croyons donc qu'il n'y a réellement pas de doute sur le caractère "frison" du trésor de Zelzate; il appartenait très probablement à l'un de ces marchands frisons, si célèbres dans l'histoire économique carolingienne.

(36) Cf. Ligees Frisioxum, ed. Richthofen (M.G.H., LL, III) pp. 632-5. 